\section{A CONTRIBUTION TO THE STUDY OF APHASIA.}

A CASE IN WHICH COMPLETE DESTRUCTION OF BROCA'S CONVOLUTION BY A NEOPLASM IN A RIGHT-HANDED PERSON PRODUCED NO APHASIA.

BY JAMES S. COLLIER, M.D., B.Sc., M.R.C.P. LoND., SENIOR HOUSE PHYSICIAN TO THE NATIONAL HOSPITAL FOR THE PARALYSED AND EPILEPTIC, QUEEN-SQUARE, BLOOMSBURY.

THE following case is of great interest as it exemplifies the activity of the glosso-kinæsthetic centre in the right rather than the left third frontal convolution in a righthanded person. I am indebted to Dr. Bastian for permission to publish the notes of this case and to Dr. Risien Russell for the pathological report.

A woman, aged 23 years, unmarried, was admitted on May 23rd, 1897, to the National Hospital for the Paralysed and Epileptic as an out-patient under the care of Dr. James Taylor. There was no family history of fits or nervous disease; her mother had died from cancer. Her previous health had always been good, with the exception of an attack of pleurisy three years before her admission to the hospital. There was no history of infantile convulsions and she had never had any trouble with her speech. She wis right-handed and wrote with her right hand, and she was skilful in playing the mandoline. No members of the family had been known to be left-handed. The history of the patient's illness was as follows. In May, 1896, when she was walking out with her sister she suddenly fell down and was unconscious for a few mi hes. There was no warning of the attack. Her face was pale but she was not convulsed, nor did she bite her tongue or pass urine during the fit. During the next fortnight she had two fits of a similar nature, but in the second fit the teeth were clenched and she was slightly convulsed all over and foamed at the mouth. Since that time she had no more fits, but she had frequent sudden attacks of giddiness, pallor, and faintness. No speech defect was noticed after any of these attacks. On examination Dr. Taylor found no signs of organic disease. The patient was treated with bromides and the attacks of giddiness became much less frequent. She regularly came to the out-patient department for the next 18 months, during which time her health was good and she continued her work. During the month of October, 1898, she was noticed to be a little dull mentally and she complained sometimes of slight headache. On Nov. 8th she was noticed by her sister to mumble in her speech and to use wrong words for the period of half an hour. For instance, she called the fire register in her bedroom the "directory" and spoke of the incandescent gaslight as the "metropolitan gaslight." This affection of speech completely passed off in half an hour and was not again noticed. On Nov. 12th she went to a theatre apparently in excellent health and on returning home she entertained her family by singing and playing the mandoline. The next morning she was noticed to be very heavy and somnolent, but she did not complain. This somnolence deepened and she was admitted into the National Hospital for the Paralysed and Epileptic as an in-patient on Nov. 14th and was placed under the care of Dr. Bastian.

On examination the patient was found to be a wellnourished, healthy-looking, rosy-faced girl. She was evidently very ill physically and she lay in bed in a semicomatose state and never moved a muscle unless roused. Her breath was offensive and her tongue was furred. When she was roused she kept on saying "Oh dear" in a sighing voice. she obeyed simple orders and answered simple questions if they were repeated several times. During the examination she said spontoneously, "Why can't you leave me alone? Let me go to sleep, I'm all right." There was no wordblindness and she could write her name, but required continuous exhortation during the process. She could only be induced to take liquid food with great difficulty. There was no hemianopia. The optic discs showed recent optic neuritis and the surface of either disc was seen with a +3 dioptre lens. There was complete palsy of the right sixth nerve and nearly complete palsy of the left sixth nerve. The left lower face did not move quite so well as the right. Motion and sensation in the trunk and limbs were natural. There was neither headache nor cranial tenderness. The knee-jerks were not exaggerated, the left being rather the more easily obtained of the two. The wrist and elbow jerks were brisk and equal. The plantar reflexes showed a flexor response on either side. Examination of the thorax and abdomen showed no abnormalities. After her admission into the hospital the stupor gradually deepened and the breathing became stertorous. The temperature ranged between $100^{\circ}$ and $102^{\circ} \mathrm{F}$. She died from respiratory failure seven days after admission without the manifestation of any further physical signs. A pathological examination was made by Dr. Risien Russell.

The necropsy was performed 12 hours after death. On removing the brain the dura mater was found adherent to the anterior part of the left temporo-sphenoidal lobe over an area of the size of a threepenny-piece. The leptomeninges: at the base of the brain showed considerable old patchy thickening. There was slight recent meningitis over the posterior extremities of the second and third left frontal convolutions, and over the anterior part of the left temporosphenoidal lobe. The cortex of the posterior end of the third frontal convolution, including that part between the ascending and posterior limbs of the Sylvian fissure and the

FIG. 1.

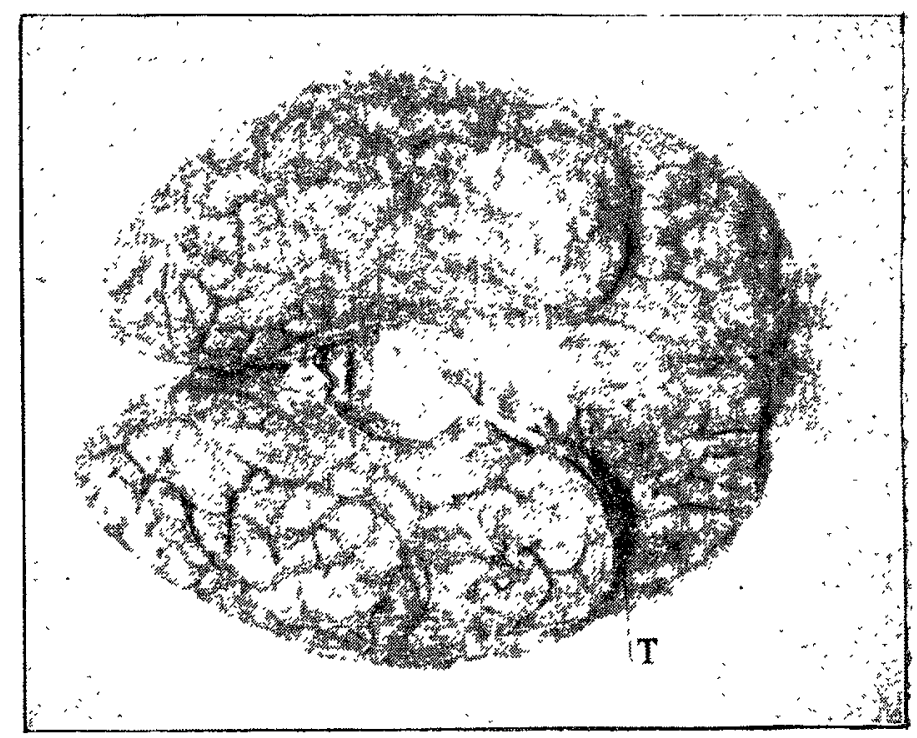

Inferior aspect of the brain, the cerehellum and pons having been removed. (The distortion of the right occipital lobe was the result of pressure during the process of hardening. T. Tumour projecting in Sylvian tissure.)

contiguous part of the left temporo-sphenoidal lobe, presented a grey jelly-like aspect suggesting the presence of a glioma in that region. To the touch this part of the cortex was elastic and yielding, suggesting the presence of a cystic condition. The inferior aspect of the brain showed a growth filling up the left Sylvian fissure and growing from the posterior part of the orbital lobule. Its projecting part was

FIG. 2.

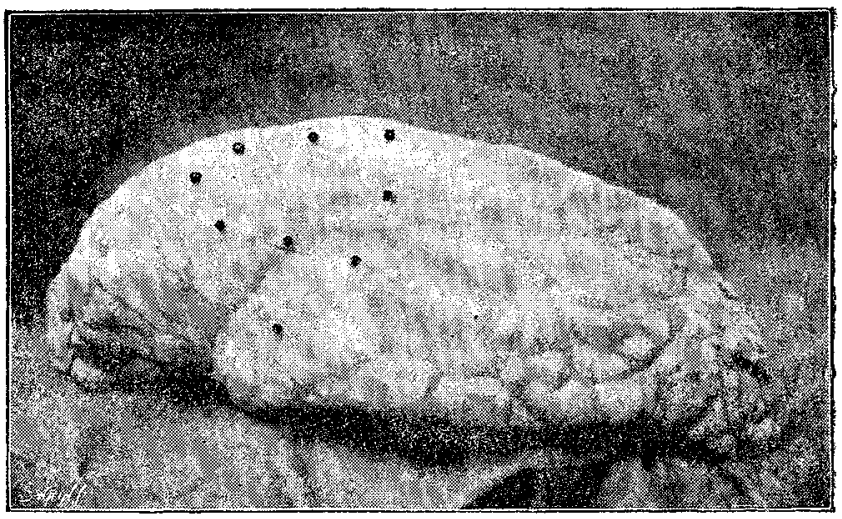

Lateral aspect of the left hemisphere. The surface-extent of the growth has been indicated by black-headed pins.

semilunar in shape, about an inch in its transverse extent and a third of an inch in its antero-posterior extent. The anterior border of the growth was parallel to the anterior border of the left temporo-sphenoidal lobe and it extended in the Sylvian fissure from close outside the outer root of the left olfactory tract to the bifurcation of the Sylvian fissure. Figs. 1, 2, and 3 show the position of the growth upon the surface of the brain. 
The brain was hardened in formalin and subsequently two horizontal sections were made, one at the level of the corpus callosum and the other at the level of the upper border of the anterior commissure. The growth was found to involve the inferior margin of the left second frontal convolution at about its middle, the whole posterior part of the left third frontal convolution, the posterior part of the orbital lobule, and to a small extent the anterior gyrus of the island of Reil. It crossed the posterior limb of the Sylvian fissure about a centimetre from its commencement and involved the anterior part of the first left temporo-sphenoidal convolution. The measurement of the growth in its maximum vertical

\section{FIG. 3.}

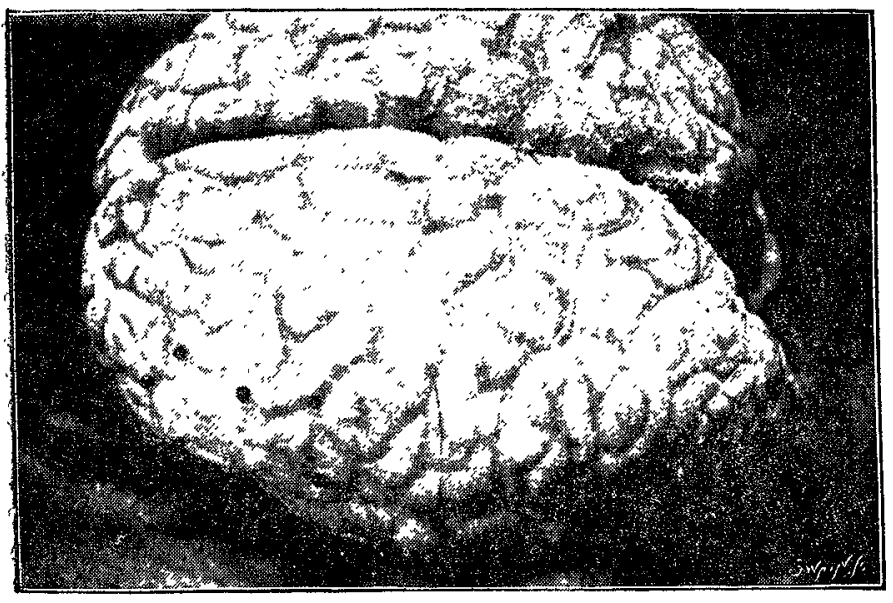

The left hemisphere seen frum the side and above. The position of the growth is indicater by hlack-headed pins. A, Fissure of Rolando.

extent was five centimetres, in its coronal extent it was 3.7 centimetres, and in its sagittal extent it was three centimetres. The ascending frontal convolution was not involved. The right third frontal convolution and the cight hemisphere appeared perfectly healthy. The growth presented a gelatinous semi-transparent appearance on section, and there were several small cysts with clear jelly-like contents of from two to four millimetres in diameter in its upper part. There were several hard white nodules in the growth, the largest being of the size of a pea. For some

FrG. 4.

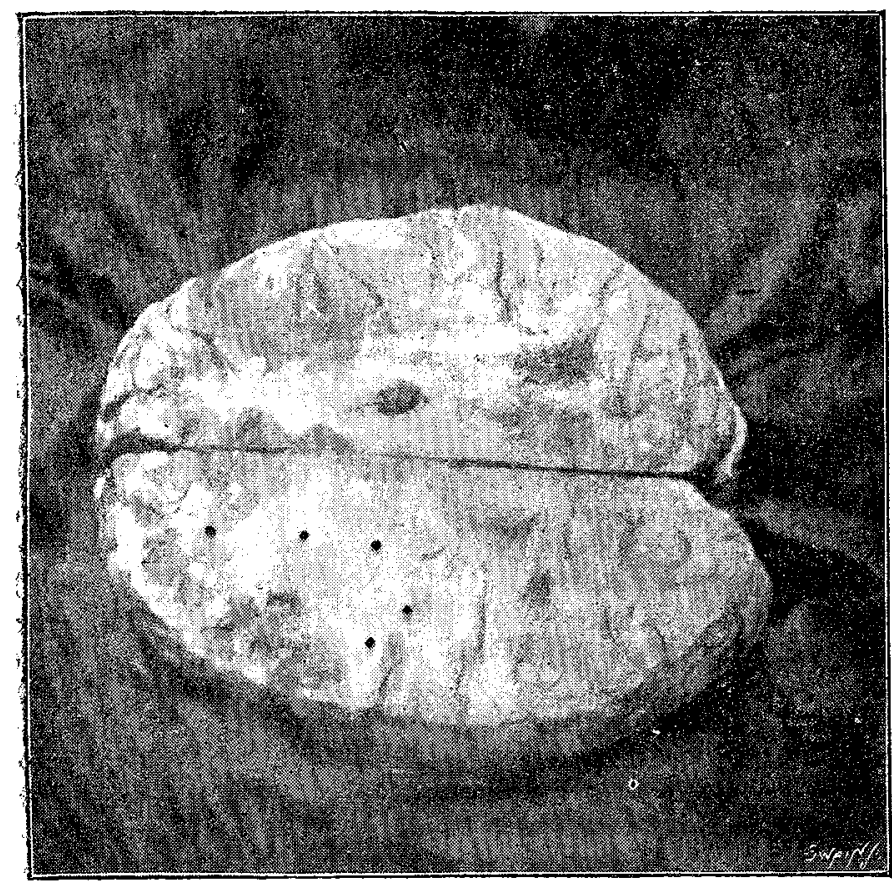

Horizontal section of the brain at the level of the corpus callosum. The area of softening round the growth is indicated.

distance round the growth the white matter of the centrum ovale was much softer than elsewhere. The extent of this condition is shown in Fig. 4. The other organs of the body presented a normal appearance. On microscopical examination the growth was found to consist of small round-celled tissue, the cells being densely aggregated around the bloodvessels which were greatly thickened. Throughout the tissue were numerous large cells which stained very faintly. Several large patches of substance presenting a caseous appearance and which did not stain were seen in each section and corresponded with the hard nodules seen with the naked eye. The appearances suggested a long-standing and slowlygrowing neoplasm.

So far as I am aware no case of the same kind as this one has been previously recorded. It proves the possibility of the fortuitous major activity of the glosso-kinasthetic centre in the right third frontal convolution in a right-handed person. It also shows that right-handedness does not necessarily involve the major activity of the third left frontal convolution for the production of articulate speech.

It has been suggested by Byrom Bramwell ${ }^{1}$ that in some right-handed persons whose ascestors were left-handed the glosso-kinæsthetic centre may possibly be situated in the right hemisphere. I obtained in this case a most complete account of the family history and there was no instance of left-handedness in the family. The presence of a pathological condition in the usual position of the glossokinæsthetic centre (the left third frontal convolution), together with a completely functioning glosso-kinæsthetic centre in another part of the brain (the right third frontal convolution), strongly suggests a causal relationship between the two. It may be argued that in this patient in early life the left third frontal convolution was the seat of some developmental or acquired defect which caused the right third frontal convolution to take up the speech function when that was learnt and which predisposed to the occurrence of the new growth, but there was no pathological evidence in support of this view. Such a lesion in early life would in all probakility have caused some atrophy of the affected cortex and asymmetry of the convolutions and in this case the convolutions were quite symmetrical upon the right and left sides. Nor was there any evidence of any trouble with the speech function in early life. She had begun to talk at the age of 18 months and had learnt to talk quickly and well. It is inconceivable that the glosso-kinæsthetic function could have been taken up by the right third frontal convolution pari passu with the devolution of the left third frontal convolution owing to the presence of a slowly growing neoplasm without some speech defect being at any time noticeable. The history of the patient, both previously to and during her illness, was obtained in the greatest detail from those who were constantly with her and no speech defect had been noticed. The facts of the case suggest that the unusual location of the leading or active glosso-kinæsthetic centre and the presence of a neoplasm in the left third frontal convolution were simply coincident, and that the right third frontal convolution had in this patient always performed the function of a glosso-kinæesthetic centre.

Left-handedness does not always transfer the leading speech centres to the right side of the brain, as exemplified in Dickinson's ${ }^{2}$ case and Wadham's ${ }^{3}$ case, and notwithstanding the immense influence of heredity, right-handedness may in rare cases be associated with leading speech centres in the right hemisphere, as I think this case proves. Bastian $^{4}$ has suggested that a similar location of the glossokinæsthetic centre may have been present in Barlow's ${ }^{5}$ case, in which after an attack of right hemiplegia and aphasia the power of speech returned on the tenth day and the patient had apparently recovered from his symptoms in a month. A second attack three months later of left brachio-facial monoplegia deprived him entirely of his speech. Symmetrical lesions of about the size of a shilling were found in each hemisphere involving the posterior end of the third frontal convolutions. In a case recorded recently by Byrom Bramwell, ${ }^{6}$ a similar explanation would accord very well with the facts of the case. In discussing his case he inclines to a different explanation. Thus he suggests " that in very rare instances, of which the present case is an illustration, the functional activity and educational endowment of the right third frontal convolution are so perfect that the speech function can be immediately taken up when the left motor vocal speech centre is acutely and completely destroyed." $\mathbf{M y}$

1 Lectures on Aphasia, Edinburgh Medical Journal, 1897, vol. ii.

2 Bastian: Aphasia and other Speech Defects, 1898, p. 90 .

3 St. George's Hospital Reports. 1868, vol. iv., p. 245. Loc. cit., p. 322

5 Brit. Med. Jour., July 28 th, 1877. o Brain, 1898, p. 343. 
suggestion is only one step farther than this and renders the explanation of the case less difficult. I would suggest that the transient speech defects in these two cases were of the same nature as are the speech defects sometimes seen in cases of left hemiplegia in right-handed persons.

The incompleteness of the notes upon speech defect in this case is due to the fact that it did not present itself as a case of aphasia. The handwriting was tested, as it is a routine practice in the National Hospital for the Paralysed and Epileptic for each patient to write his name in the notes. Word vision was tested in the routine examination of eyes presenting optic neuritis.

\section{A PRELIMINARY NOTE UPON CERTAIN ORGANISMS ISOLATED FROM CANCER AND THEIR PATHOGENIC EFFECTS UPON ANIMALS. ${ }^{1}$}

BY H. G. PLIMMER, M.R.C.S. ENG., F.L.S., PATHOLOGIST AND LECTURER ON PATHOLOGY AND BACTERIOLOGT, ST. MARY'S HOSPITAL.

DURING the past six years I have been studying the cellinclusions which are found in cancer and their relation both to the origin and course of the disease, and for this work I have had to examine 1278 cancers taken from various organs and parts and of all possible varieties. Out of this large number of cases there have been a few-nine in all-in which the cell-inclusions have been extremely numerous, so that at the growing edge, and even far into the tumour, scarcely a cell could be found without an inclusion, sometimes with as many as 36 even of these inclusions in one cell, and these bodies have been similar to those which Metchnikoff, Ruffer, and others, as well as myself, have regarded and described as parasites standing in causal relationship to the disease. In two out of the nine cases mentioned these bodies have been present in enormous numbers, and I have succeeded in isolating from the last of these remarkable cases an organism which is pathogenic in a peculiar manner to certain animals and whose virulence I have been able to keep unimpaired for some months.

Previous Work on the Experimental Production of TUMOURS IN ANIMALS.

The only work, I think, that needs mention here in connexion with this heading is that of Sanfelice in Cagliari and of Roncali in Rome. Sanfelice has produced tumours in animals with organisms which he isolated from infusions of various fruits, and they both have isolated organisms from cancers, which I believe from their descriptions-I have not seen their cultures-are morphologically somewhat similar to those I am about to bring before you. But Sanfelice's organism appears to have been very difficult to isolate in a virulent form from human cancer and to keep virulent, so that in his last paper ${ }^{2}$ he treats only of the organisms derived from fruit infusions and of their effects upon animals. Most of the statements are doubted by the German pathologists, including such a good observer as Baumgarten, but from my own experience $\mathrm{I}$ do not find any reason to doubt any of Sanfelice's statements, and I think that he deserves the greatest credit for removing the study of the etiology of cancer from the histological to the experimental region of work.

\section{ON THE METHOD OF ISOlation ADOPTED.}

The cancer from which the organisms I am about to describe were isolated and with which my experiments have been made was taken from the breast of a woman, aged 35 years ; it had a history of only two months' duration and it was growing rapicly at the time of the operation. Immediately after removal I examined a fresh scraping and finding such an extraordinary number of the bodies $I$ have mentioned in the cells $I$ cut, with all possible precautions against contamination, with a carefully sterilised knife, very thin slices from the growth which I placed with a little of the juice scraped from the cut surface in a flask containing the following liquid, which was of course carefully sterilised.

1 A paper read before the Roval Society on March 9th, 1899 ; communicated by Professor J. Rose Bradford, F.R.S.
2 Zeitschrift fïr Hygiene, 189
This medium consisted of an infusion made from cancer, just as the ordinary beef infusion is made, to which was added, after careful neutralisation, 2 per cent. of glucose and 1 per cent. of tartaric acid. This medium was the outcome of many trials with all kinds of mixtures and $I$ tried it in this case as I had already got similar organisms to grow in it from two previous cases but they had no pathogenic properties, and this, I think, was due to the omission of the next step. This medium, too, is particularly useful, as hardly any bacteria, however hardy, will grow upon it. Then. remembering that in the body these organisms were under anaerobic conditions, I exhausted the air from my flasks and passed hydrogen into them and finally sealed them up. This I have found is of great importance as regards the maintenance of the virulence, and $I$ find consequently that there is no falling off in the virulence of my cultures which are as active now as they were four months ago. Five flasks werc made in this way, but in spite of precautions two became contaminated with moulds; in the other three, however, I got after from three to five days a pure culture of the organism described below has been kept growing in this and various other media ever since.

Morphology and Relation to Media.

The organism is apparently a saccharomyces, but $I$ arw informed that, according to some authorities, such as De Bary, Cuboni, and Duclaux, the saccharomycetes are. nothing but the developmental stages of fungi which really belong to either the phycomycetes, the ascomycetes, or the basidiomycetes. Moreover, they state that in some species of mycelium-forming fungi, single parts, especially conidia, car grow in the saccharomyces form on certain nutrient media, so I will not attempt to locate this organism at present. Sanfelice and Roncali, however, definitely state that the organisms they have isolated are blastomycetes.

When grown in the medium described these organisms produce a cloudiness which becomes visible in about 48 hours and increases till about the sixth day, when the growth sinks to the bottom, the medium then becoming clear: $n c^{\text {a }}$ scum or pellicle is formed. When grown on this medium. solidified with agar the organisms form small round colonies: which remain separate. After some weeks the colour, which was originally white, becomes yellow. The colonies do not attain at any time a great size. ${ }^{3}$ Gelatin is not liquefied, but the growth on this medium is never luxuriant. On potato a thick white layer is formed which in about two weeks will cover the entire surface, changing then to a yellowish-brown colour. They will grow aerobically, but not so well, at any rate at first, and they lose their virulence in a short time if continuously grown in this way. Microscopically they are round bodies, frequently growing in clumps, with a central portion which stains deeply, and in most cases with a thin, strongly refractile capsule which sometimes shows a double contour; but some young forms can be seen which are apparently without a capsule. The size varies from 0.004 millimetre to 0.04 millimetre. Their reproduction. appears to be by budding, but I have fancied that I have also seen in a few instances endogenous budding; of this, however, I am not certain. These bodies correspond morphologically with those found in the original tumour and also with those described by Ruffer and myself and by some others of those who have worked at the microscopical appearances of cancer.

\section{ExPERIMENTAL RESULTS.}

I have selected from the experimental work which I have done with these organisms those experiments which seemed to me to be the most important. Up to the present time I have not been able to make any such experiments upon such animals as would allow of the easy bringing of these organisms into contact with a likely epithelial surface with the exception of the cornea (vide Experiment No. 4), but through the kindness of Dr. Rose Bradford I have beer enabled now, at the Brown Institution, to inoculate a bitch in the mammæ, but the time is as yet too short to enableme to make any statement as to the result. The cultures used in the following experiments were made in the medium. previously described.

1. Rabbit.-Intravenous injection of five cubic centimetres of an eight days old culture. There was no obvions result.

3 The following specimens were shown: (1) sections of the cancer from which the cultures were made; (2) the cultures on various media : (3) preparations of the cultures; (4) sections of the organs of the animals in which tumours har been produced; and (5) animals, os portions of them, in which tumours had been produced. 